\title{
A nanowell-based QCM aptasensor for rapid and sensitive detection of avian influenza virus
}

\author{
Ronghui Wang ${ }^{\mathrm{a}}$, Lijun Wang ${ }^{\mathrm{a}, \mathrm{b}}$, Zachary T. Callaway ${ }^{\mathrm{a}}$, Huaguang Lu ${ }^{\mathrm{c}}$, Tony Jun Huang ${ }^{\mathrm{d}}$, and \\ Yanbin $\mathrm{Li}^{\mathrm{a}, \mathrm{e}^{*}}$
}

${ }^{a}$ Department of Biological and Agricultural Engineering, University of Arkansas, Fayetteville, AR 72701, USA

${ }^{\mathrm{b}}$ College of Life Science and Food Engineering, Nanchang University, Nanchang, 330031, China

${ }^{c}$ Wiley lab, Department of Veterinary and Biomedical Sciences, Pennsylvania State University, University Park, PA 16802, USA

d Department of Engineering Science and Mechanics \& Material Research Institute, Pennsylvania State University, University Park, PA 16802, USA

${ }^{\mathrm{e}}$ Center of Excellence for Poultry Science, University of Arkansas, Fayetteville, AR 72701, USA

* Corresponding author: Department of Biological and Agricultural Engineering, University of Arkansas, 203 Engineering Hall, Fayetteville, AR 72701, USA. Tel.: +1 4795752881; fax: +1 4795752846. Email address: yanbinli@uark.edu 


\begin{abstract}
In this study, a cost-effective nanowell structure was fabricated and utilized for the development of a nanowell-based quartz crystal microbalance (QCM) aptasensor for rapid, sensitive, and label-free detection of H5N1 avian influenza virus (AIV). A nanoporous gold film with a thickness of $120 \mathrm{~nm}$ and a pore size of $\sim 20 \mathrm{~nm}$ was prepared using a metallic corrosion method. Then, the nanoporous gold film was immobilized onto a gold electrode surface using a self-assembled monolayer to form a nanowell-based electrode. A specific H5N1 AIV ssDNA aptamer with a $\mathrm{NH}_{2}$ conjugated 5'-terminal was used in the fabrication of the QCM aptasensor through covalent bonding. The stepwise assembly of the aptasensor was characterized by means of QCM. The result showed that the binding of target AIV H5N1 onto the immobilized aptamers decreased the sensor's resonant frequency, and the frequency change correlated to the virus titer. We demonstrated that the developed nanowell-based QCM aptasensor could dramatically reduce detection time down to $10 \mathrm{~min}$ using a label-free assay. The detection range of $2^{-4}$ to $2^{4}$ hemagglutination units (HAUs) $/ 50 \mu 1$ was obtained with a detection limit of $2^{-4}$ HAU/50 $\mu 1$ for AIV H5N1. The binding of target H5N1 virus onto the nanowell-based electrode surface was further confirmed by scanning electron microscopy (SEM). No interference was observed from non-target AIV subtypes of H1N1, H2N2, H7N2 and H5N3. The aptasensor using H5N1 aptamer was validated for the detection of AIV H5N1 in chicken tracheal swab samples. The developed aptasensor could be adopted for detection of other viruses.
\end{abstract}

Keywords: nanowell-based electrode, quartz crystal microbalance, aptasensor, virus detection, avian influenza 


\section{Introduction}

In recent years it has been proven that electrode material and structure play critical roles to achieve simple, sensitive, rapid, and stable detection in bioanalysis using electrochemical or piezoelectrical methods [1-3]. Nanomaterials and three-dimensional (3D) nanostructures have been introduced into electrode structures to improve the performance of electrical measurements [4-6]. In particular, nanowell-structured surfaces have been attractive in the immobilization of biomolecules [7-9] since they can provide a nanowell pattern that greatly increases surface area. The nanowell-structured surfaces can make the capacity and density of ligand immobilization much higher than those on surfaces without nanowell structures. There are some published studies using the fabrication of nanowell structures and the binding of biomolecules onto nanowell surfaces [9-11], but very little focus on the development of a nanowell-based biosensor for practical application. There are a couple of studies that reported the analysis of DNA hybridization using well-oriented nanowell arrays [8] and nanowell array electrodes [12]. The results showed a two-orders-of-magnitude enhancement in sensitivity [8], which demonstrated that the nanowell-based nanostructure could result in an increased sensitivity. Taking advantage of the nanowell structure, in this study, a nanowell-based QCM aptasensor was developed for rapid, sensitive, and label-free detection of AIV H5N1.

AIV infections or outbreaks have continued to have a large impact on the poultry industry and also represent a threat to human health. The highly pathogenic avian influenza (HPAI), H5N1, which originally emerged in southeast Asia in the late 1990's cost the poultry industry an estimated \$10 billion between 1997 and 2008 [13]. The HPAI H5N1 has also caused global concerns for public health and continues to spread throughout the world. Since 2003, a total of 62 countries (regions) have been affected by HPAI H5N1, and new cases of human and animal 
infections continue to be reported [14]. The AIV transmission link between humans and animals has given rise to concern among public health authorities and the scientific community regarding the pandemic potential of HPAI outbreaks. The H5N1 AIV has caused 850 human infections and 449 deaths since 2003, according to the World Health Organization (WHO) [14]. Therefore, advanced technologies for more rapid and sensitive AIV detection are needed for better surveillance and effective control of AIV outbreaks.

Commonly available AIV detection techniques include virus isolation, serological ELISA, antigen-capturing, and polymerase chain reaction (PCR)-based assays [15-17]. Virus isolation has been the "gold standard" as it is the highest in sensitivity and reliability, however, it is a very time-consuming procedure (3-7 days). Serological ELISA is a rapid test that can use mass serum samples and is commonly used for serum antibody detection, but it is not used for virus detection in practice or diagnostics. The molecular assays of the RT-PCR and real-time RT-PCR methods have the advantages of high sensitivity and specificity, but possess disadvantages of requiring expensive PCR equipment and reagents as well as technically skilled personnel. As an alternative, efforts have been directed towards the development and establishment of a novel biosensor technique for the rapid and effective detection of AIVs. Various biosensors have been reported, such as surface plasmon resonance (SPR), QCM, fluorescence, optical, imaging ellipsometry, interferometric, and electrochemical [18-32]. Among them, QCM biosensors have attracted interest in applications for AIV detection due to their simplicity, cost-effectiveness, direct detection, and real-time output [28-32]. In order to improve sensitivity, a mass-amplified QCM assay is usually constructed using a sandwich format, in which micro/nanoparticles are used as "mass enhancers" to amplify the detection signal [30]. However, this amplification step would result in a prolonged detection time. 
In this study a cost-effective nanowell structure was fabricated and utilized for the development of a nanowell-based QCM aptasensor for rapid, sensitive, and label-free detection of AIV H5N1. The nanowell-based electrode served as a 3D matrix for ligand (aptamer) immobilization, which resulted in a 5-fold increase in the amount of ligand immobilized when compared to the electrode without nanowells. The developed QCM aptasensor was able to directly detect target viruses with a dramatically reduced detection time (10 min) without the need of labels or "mass enhancers", and a detection limit of $2^{-4}$ hemagglutination unit (HAU) /50 $\mu 1$ for AIV H5N1 was obtained. Non-target AIV subtypes (e.g., H1N1, H2N2, H7N2 and H5N3) were used to test the specificity of the aptasensor. Scanning electron microscopy (SEM) was used to confirm the binding of AIV H5N1 on the nanowell-based electrode surface. Finally, tracheal swab samples from chickens were used to evaluate the QCM aptasensor.

\section{Materials and Methods}

\subsection{Materials and reagents}

16-Mercaptohexadecanoic acid (MHDA), 1,6-hexanedithiol (HDT), N-(3dimethylaminopropyl)-N'-ethylcarbodiimide hydrochloride (EDC.HCL), N-hydroxysuccinimide (NHS), Poly (ethylene glycol) methyl ether thiol (PEG), and phosphate buffered saline (PBS, 0.01 M, pH 7.4) were purchased from Sigma-Aldrich (St. Louis, MO). PEG was dissolved in 10 $\mathrm{mM}$ PBS at a concentration of $0.1 \mathrm{mg} / \mathrm{ml}$ and prepared fresh for each test. Ultrapure water (18 $\mathrm{M} \Omega \cdot \mathrm{cm}$ ) produced by a Millipore-Milli-Q system (Bedford, MA) was used throughout each test. $12 \mathrm{~K}$ white gold leaf sheets $(51.0 \% \mathrm{Au}, 48.0 \% \mathrm{Ag}, 1.0 \% \mathrm{Pd}$, thickness of each leaf: $120 \mathrm{~nm})$ were obtained from LA Gold Leaf Wholesaler (Covina, CA). 
Inactivated H5N1 AIV (Scotland/59) was supplied from the USDA/APHIS National Veterinary Services Laboratories (NVSL, Ames, IA). The stock concentration of H5N1 virus was $128 \mathrm{HAU} / 50 \mu 1$. Other AIV subtypes H1N1, H2N2, H7N2, and H5N3 were prepared at Wiley Lab at Pennsylvania State University, University Park, PA. The selected aptamer with high affinity and specificity against AIV H5N1 was developed in our group with detailed information described in our previous study [41]. $\mathrm{NH}_{2}$ conjugated aptamer was obtained from Integrated DNA Technologies, Inc. (Coralville, IA). The sequence was: 5'- $\mathrm{NH}_{2}$ - $\mathrm{C}_{6}$-GTG TGC ATG GAT AGC ACG TAA CGG TGT AGT AGA TAC GTG CGG GTA GGA AGA AAG GGA AAT AGT TGT CCT GTT G-3'.

Chicken tracheal swab samples were used in this study. Three swabs were pooled and placed into a sterile tube containing $3 \mathrm{ml}$ of PBS. To process the swabs for testing, each swab was vortexed vigorously for $15-20 \mathrm{~s}$ and then pressed against the inside of the tube to squeeze and wash off as much of the organic material as possible. The liquid swab samples were kept at $4^{\circ} \mathrm{C}$ in a refrigerator for $1 \mathrm{~h}$ to allow large particles and organic materials to precipitate to the bottom of the sample tube. The supernatants of samples were then stored at $-80^{\circ} \mathrm{C}$ in a freezer until used. Before QCM testing, the swab samples were thawed at room temperature and filtered through a $0.45 \mu \mathrm{m}$ syringe filter. The filtrate was spiked with the inactivated H5N1 AIV in different titers of HAU to be ready for aptasensor detection.

\subsection{Instruments and quartz crystals}

The circular AT-cut $7.995 \mathrm{MHz}$ quartz crystals with Au electrodes evaporated on both sides were purchased from International Crystal Manufacturing Co. (Oklahoma City, OK, USA). The quartz crystals were $14 \mathrm{~mm}$ in diameter and $0.2-\mathrm{mm}$ thick and the gold electrodes were 5.1 $\mathrm{mm}$ in diameter and 100-nm thick. The quartz resonator was housed inside a methacrylate cell 
(International Crystal Manufacturing Co. OK, USA) so that only one side of the crystal was in contact with the solution in the cell well. The frequency variations were continuously recorded using a QCA922 quartz crystal analyzer (Princeton Applied Research, Oak Ridge, TN).

\subsection{Preparation of the nanoporous gold film}

The nanoporous gold film was prepared using the metallic corrosion method [33]. Briefly, it was prepared by floating $12 \mathrm{~K}$ white gold leaves on concentrated $\mathrm{HNO}_{3}$ solution for 60 min, and then subsequently rinsing them with MilliQ water three times. During this process,

nearly all Ag atoms were dissolved away, leaving behind a bicontinous porous Au membrane structure with a pore size of around $20 \mathrm{~nm}[34]$.

2.4 Immobilization of the nanoporous gold film onto the gold electrode surface to form a nanowell-based electrode

The quartz resonator was cleaned by dipping it in $1 \mathrm{M} \mathrm{NaOH}$ for $20 \mathrm{~min}$ and $1 \mathrm{M} \mathrm{HCl}$ for 5 min in sequence. It was then thoroughly washed with MilliQ water and dried in a stream of nitrogen. The nanoporous gold film was floated on MilliQ water and then was attached to the cleaned quartz resonator and allowed dry for $12 \mathrm{~h}$ at room temperature. Next, the quartz resonator attached with nanoporous gold film was immersed in a mixture solution of $20 \mathrm{mM}$ MHDA (in ethanol) and 1\% HDT (in ethanol) at a ratio of 1:1 for $24 \mathrm{~h}$ at room temperature in the dark. After rinsing with ethanol (99\%) and water, the nanowell-based electrode was formed and was ready for aptamers immobilization.

\subsection{Immobilization of aptamers onto the nanowell-based gold electrode surface}


The pretreated nanowell-based electrode was fixed into the detection cell and connected to the quartz crystal analyzer. First, $200 \mu \mathrm{l}$ of MilliQ water was added in the cell well to get a baseline. Second, $200 \mu \mathrm{l}$ EDC/NHS (75 mM/30 mM, v/v, 1:1) solution was dropped into the cell well (10 min) for activation. Third, after MilliQ water washing, $100 \mu \mathrm{l}$ of $\mathrm{NH}_{2}$-aptamer $(1 \mu \mathrm{M})$ was added and incubated for $30 \mathrm{~min}$. Then, $200 \mu \mathrm{l}$ of the blocking agent PEG $(0.1 \mathrm{mg} / \mathrm{ml})$ was added for 25 min to prevent any nonspecific binding to the electrode surface. Finally, after washing with PBS buffer (0.01 M, pH 7.4), the aptamer immobilized electrode was ready for AIV detection.

\subsection{AIV detection}

A series of 2-fold dilutions of the target AIV H5N1 were prepared, and $200 \mu \mathrm{l}$ of each dilution was added into the detection cell and incubated for $10 \mathrm{~min}$ at room temperature. After incubation, the nanowell-based electrode was washed with PBS (300 $\mu 1$ per wash) three times. The frequency change $(\Delta F)$ caused by AIV binding was calculated by measuring the difference before and after adding the target virus. After the binding of target virus, the sensor surface was regenerated with $1,2,5$, or $10 \mathrm{mM} \mathrm{NaOH}$ solution to regenerate the aptamer surface for a new binding reaction.

\subsection{SEM images}

SEM imaging was conducted to confirm the binding of AIV H5N1 onto the nanowellbased electrode gold surface using the high-resolution scanning electron microscope FEI Nova NanoLab 200 (FEI Company, Hillsboro, Oreg.) in field immersion mode at $30 \mathrm{kV}$ accelerating voltage. Samples for the SEM were prepared by following the electrode immobilization and virus binding protocol and allowing the samples to air dry in a fume hood. 


\subsection{Statistical analysis}

Microsoft Excel (Microsoft, Redmond, VA) was used for the statistical analysis of all data, including calculation of means, standard deviations, and t-tests. Means were considered significantly different at $P \leq 0.05$.

\section{Results and Discussion}

\subsection{Fabrication and characterization of the nanowell-based QCM aptasensor}

The nanoporous gold film was prepared using the metallic corrosion method [33] reported by Ding and Erlebacher, who found that the silver could be selectively dissolved using nitric acid on silver/gold alloys to generate a nanoporous gold film. By simply floating commercially available $12 \mathrm{~K}$ white gold leaf (Ag/Au alloy, 50:50 wt \%) on a concentrated nitric acid surface for $1 \mathrm{~h}$, the obtained pore size was uniform and approximately $20 \mathrm{~nm}$ when the thickness of the film was $100 \mathrm{~nm}$ [34]. The metallic corrosion method was simple and inexpensive, so the same protocol was applied to a commercially available $12 \mathrm{~K}$ white gold leaf with thickness of $120 \mathrm{~nm}$ to produce the nanoporous gold film (pore size $~ 20 \mathrm{~nm}$ ) in this study. Then, the Energy-Dispersive X-ray Spectroscopy (EDS) was used for elemental analysis on the nanoporous gold film before and after $\mathrm{HNO}_{3}$ treatment. The commercial $12 \mathrm{~K}$ white gold sheet contained $\mathrm{Ag}, \mathrm{Au}, \mathrm{Pd}$ and $\mathrm{O}$ (provided by the commercial company), so these elements were analyzed and results showed that the $12 \mathrm{~K}$ white gold sheet contained $47 \% \mathrm{Au}, 48 \% \mathrm{Ag} 1 \%, \mathrm{Pd}$ and $4 \% \mathrm{O}$ before the treatment. After the treatment, more than $90 \% \mathrm{Ag}$ (4\% remaining) and $100 \% \mathrm{Pd}(0 \%$ remaining) were dissolved away, but $\mathrm{Au}$ and $\mathrm{O}$ were increased to $87 \%$ and $9 \%$, respectively. 
To form the nanowell-based electrode, the nanoporous gold film was immobilized onto the gold electrode surface through a bifunctional dithiol HDT (1,6-hexanedithiol). The bifunctional dithiol HDT is a good molecular candidate to be used as a cross-linker because of its easy preparation, high stability, and ordered structures [35-37]. Most importantly, it can form densely packed and highly ordered films with an upright molecular structure when selfassembled on gold surfaces [35-37], and the crosslinked structure cannot be destructed by solvent or heat treatment [38]. In this study, we used a mixture solution of 1\% HDT and $20 \mathrm{mM}$ MHDA (16-mercaptohexadecanoic acid) at a ratio of 1:1 for the preparation. As shown in Fig. 1a, HDT was applied as a bifunctional cross-linker to link the nanoporous gold film with the gold electrode, forming a nanowell-based electrode. MHDA is a long-chain carboxylic acidterminating alkanethiol, which was self-assembled on the gold surface via the strong Au-thiolate bond and also provided the tail carboxylic functional group for further biomaterial immobilization (Fig. 1a). The SEM image of a nanowell-based electrode surface is shown in Fig. $1 \mathrm{~b}$, and the pictures of the QCM electrode before (I) and after (II) the nanoporous gold film modification are presented in Fig. 1c.

Specific ssDNA aptamer to AIV H5N1 was conjugated with an amino group at its 5'terminal, which was used in the fabrication of the QCM aptasensor through covalent bonding. The mechanism of the $\mathrm{NH}_{2}$-aptamer immobilization is illustrated in Fig. 2a. A monolayer of MHDA was attached to the nanowell-based electrode gold surface. The monolayer of MHDA is stable in aqueous solution at room temperature and more thermally stable than the selfassembled monolayers (SAMs) from short chain disulfides or thiols. The MHDA functionalized gold surface was then treated with EDC and NHS to form an active NHS ester. After surface activation, the NHS ester was replaced by the amino group presented on the 5 '-terminal of the 
aptamers. Therefore, the $\mathrm{NH}_{2}$-aptamers were immobilized through the amide bond. The MHDA mediated biomolecule immobilization has been demonstrated as an effective scheme for sensor surface preparation, which can lead to a highly efficient ligand-target interaction [30, 39]. Fig. 2b shows the typical temporal responses of frequency shifts for the stepwise assembly of the fabrication of the nanowell-based QCM aptasensor. Based on the SAM technique, the immobilization of $\mathrm{NH}_{2}$-aptamers resulted in a $115 \mathrm{~Hz}$ decrease of resonant frequency, indicating the successful assembly of the aptamer on the nanowell-based QCM sensor surface. Following the immobilization of aptamer on the QCM sensor surface, a freshly prepared blocking solution PEG $(0.1 \mathrm{mg} / \mathrm{ml})$ was applied to block uncoated surface sites and reduce non-specific adsorptions. Because PEG has a small size and strong blocking capability, it has been proven to be very effective not only preventing non-specific binding to the gold surface, but also by not interfering with the aptamer's ability to capture the target [31].

The gold surface area of a nanowell-based electrode was calculated based on $\sim 50 \%$ nanowells present on the surface. This was because all $\mathrm{Ag}$ was dissolved away from $\mathrm{Ag} / \mathrm{Au}$ alloy $(\sim 50: 50 \mathrm{wt} \%)$ after it was exposed to concentrated nitric acid treatment. The total gold surface area $(A)$ of an electrode without nanowells was:

$A=\pi r^{2}=3.14 \times(2.55 \mathrm{~mm})^{2}=20.4 \mathrm{~mm}^{2}$

Since the area is $\sim 50 \%$ pores, the top view of the total pore surface area $\left(S A_{\text {pore-total }}\right)$ becomes:

$S A_{\text {pore-total }}=20.4 \mathrm{~mm}^{2} \times 0.5=10.2 \mathrm{~mm}^{2}$

The top view surface area $\left(S A_{\text {pore }}\right)$ of one pore (10 nm radius) is:

$S A_{\text {pore }}=\pi r^{2}=3.14 \times(10 \mathrm{~nm})^{2}=314 \mathrm{~nm}^{2}$

After the total pore surface area and single pore surface area were found, the number of pores $(N)$ was calculated by dividing this total pore surface area $\left(S A_{\text {pore-total }}\right)$ by the surface area of a single pore $\left(S A_{\text {pore }}\right)$ : 
$N=\frac{S A_{\text {pore-total }}}{\text { SA pore }}=\frac{10.2 \mathrm{~mm}^{2}}{314 \mathrm{~nm}^{2}}=32,484,076,433$

If each pore is considered as cylindrical in shape (120 nm in height and $20 \mathrm{~nm}$ in diameter), the surface area of one pore due to the height $\left(S A_{\text {cylinder-height }}\right)$ is:

$S A_{\text {cylinder-height }}=2 \pi r h=2 \times \pi \times 10 \mathrm{~nm} \times 120 \mathrm{~nm}=7.539 \times 10^{-9} \mathrm{~mm}^{2}$

Now this number can be multiplied to the total number of pores to get the total surface area due to the cylinders height:

$7.539 \times 10^{-9} \mathrm{~mm}^{2} \times 32,484,076,433=245 \mathrm{~mm}^{2}$

Now the total surface area of the nanowell-based electrode can be found by adding this surface area due to the cylinders height to the $A$ from equation (1):

$245 \mathrm{~mm}^{2}+20.4 \mathrm{~mm}^{2}=265.4 \mathrm{~mm}^{2}$

Based on the above calculation, the total surface area of the nanowell-based electrode increases the surface area by $245 \mathrm{~mm}^{2}$ or $((265.4 / 20.4) \times 100)=1,300 \%$. During the immobilization procedure, MHDA and HDT at a ratio of 1:1 were applied, therefore, only half of the gold surface area was functionalized with carboxylic functional groups for further $\mathrm{NH}_{2}$ apatmer immobilization. This means the functionalized surface area on the nanowell-based electrode increased by 6.5 times of the normal amount without using a nanowell. To prove the enhanced capacity of ligand immobilization on the nanowell-based electrode due to the greatly increased gold surface area, we conducted a comparison test of the ligand (aptamer) immobilization using the QCM crystal electrode with and without the nanowells. The same immobilization protocol was applied to both electrodes. The immobilization of aptamers on the surface of nanowell-based electrode resulted in $-115 \pm 15 \mathrm{~Hz}$ change in frequency, which showed a fivefold increase in the amount of aptamer immobilization, when compared to the electrode without nanowells $(-23 \pm 4 \mathrm{~Hz})$. 


\subsection{Detection of AIV H5N1 using the nanowell-based aptasensor}

Following the fabrication of the nanowell-based aptasensor, the inactivated stock AIV H5N1 with an original titer of $128 \mathrm{HAU} / 50 \mu \mathrm{l}$ was diluted with PBS in serial concentrations for detection. Frequency shift caused by the target virus was used as the detection signal of this aptasensor. The total detection time was only 10 min. Fig. 3a shows the typical QCM detection curve of AIV $\mathrm{H} 5 \mathrm{~N} 1$ at a titer of 2 and $8 \mathrm{HAU} / 50 \mu \mathrm{l}$. A clear decrease in resonance frequency shift was observed for detection of both titers, and the corresponding response in resonance frequency was -116 and $-176 \mathrm{~Hz}$, respectively, indicating that the developed nanowell-based QCM aptasensor was capable of detection of target AIV H5N1. Next, the AIV H5N1 with titers in the range of $2^{-4}-2^{4} \mathrm{HAU} / 50 \mu \mathrm{l}$ were tested using the nanowell-based aptasensor and the results are shown in Fig. 3b. Triplicate tests were done for each measured titer, and the standard deviations (S.D.) are shown as error bars in the figure. A linear relationship was found in the AIV H5N1 titer range from $2^{-4}$ to $2^{0} \mathrm{HAU} / 50 \mu \mathrm{l}$, and then again from $2^{0}$ to $2^{4} \mathrm{HAU} / 50 \mu \mathrm{l}$. The equation $\mathrm{y}=7.1 x+35.4\left(R^{2}=0.96\right)$ corresponded to the range of $2^{-4}-2^{0} \mathrm{HAU} / 50 \mu \mathrm{l}$, and $\mathrm{y}=51.3 x+38.2\left(R^{2}=0.97\right)$ to the range of $2^{0}-2^{4} \mathrm{HAU} / 50 \mu \mathrm{l}$, respectively, where $y$ was the decrease in frequency (Hz), and $x$ was AIV H5N1 titer expressed in $\log _{2}{ }^{\mathrm{HAU} / 50 \mu \mathrm{l}}$. PBS without target virus was also detected as a negative control (blank), and a frequency shift of $2 \pm 2 \mathrm{~Hz}$ was obtained. The threshold for the positive detection [30] was set as background (blank) $+3 \times$ noise (standard deviation) $=8 \mathrm{~Hz}$, and the detection limit of the nanowell-based QCM aptasensor was determined as $2^{-4} \mathrm{HAU} / 50 \mu \mathrm{l}$, which was comparable to the previously reported QCM immunosensor [30] and the hydrogel based QCM aptasensor [32]. However, the reported QCM immunosensor required a signal amplification with nanobeads and the detection time was $2 \mathrm{~h}$. The nanowell-based QCM aptasensor developed in this research was a label-free detection 
approach with a detection time of only $10 \mathrm{~min}$. Although the hydrogel based QCM aptasensor was a label-free detection method, it still required 30 min detection time.

SEM was used to further confirm the binding of the target AIV H5N1 onto the aptamer immobilized nanowell-based electrode surface. Fig. 3c shows the top view of the SEM images for the nanowell-based electrode surface after the binding of AIV H5N1. A single virus captured on the nanowell-based electrode surface was clearly observed as pictured by two different images. The size of the captured virus particle was $\sim 100 \mathrm{~nm}$, which is consistence with the diameter of a typical AI virus $(80-120 \mathrm{~nm})[26,40]$.

For comparison, a QCM crystal electrode without nanowells was subjected to the same treatment for the aptamer immobilization and then was used for detection of AIV H5N1 at the titers of $2^{-4}, 2^{-2}, 2^{0}, 2^{2}$ and $2^{4} \mathrm{HAU} / 50 \mu 1$. Triplicate tests were done for each measured titer and the result is listed in Table 1. It can be seen that a significant increase in frequency change $(P$ value $\leq 0.05$ ) was obtained by the nanowell-based aptasensor with virus titers of $2^{-2}, 2^{0}, 2^{2}$ and $2^{4}$ HAU/50 $\mu 1$ when compared to that without nanowells. When the target virus titer was $2^{2}$ and $2^{4}$ $\mathrm{HAU} / 50 \mu \mathrm{l}$, the nanowell-based aptasensor resulted in the frequency change of $-126 \pm 10 \mathrm{~Hz}$ and $259 \pm 22 \mathrm{~Hz}$, respectively. However, the aptasensor without nanowells could only generate $72 \pm 11 \mathrm{~Hz}$ and $-163 \pm 24$ change in frequency for the same virus titers of $2^{2}$ and $2^{4} \mathrm{HAU} / 50 \mu \mathrm{l}$. The result implied that the nanowell-based aptasensor had an enhanced capacity of ligand-target interactions, which was possibly due to the nanowell structure and the increased surface area.

The specificity of the developed nanowell-based aptasensor was evaluated using nontarget AIV subtypes H7N2, H1N1, H5N3, and H2N2 with a titer of $2 \mathrm{HAU} / 50 \mu \mathrm{l}$ and the result was summarized in Fig. 4a. While AIV H5N1 caused a $-105 \pm 11 \mathrm{~Hz}$ frequency change, none of the four non-target AIV subtypes caused more than $-7 \mathrm{~Hz}$ of frequency shift, which is below the 
lower detection limit of $-8 \mathrm{~Hz}$ frequency change. The result indicated that the developed nanowell-based aptasensor had good specificity for the target AIV H5N1. The specificity of a biosensor is mainly dependent upon the ligands (such as antibodies and aptamers) immobilized on the sensor surface. The selected aptamer used in this study for the fabrication of the nanowellbased aptasensor was developed and reported in our previous study, which displayed strong binding affinity and high specificity against AIV H5N1 [41].

The chicken tracheal swabs were experimentally spiked with the inactivated AIV H5N1 and then were used to test the performance of the nanowell-based aptasensor for the detection of AIV H5N1 with a complex background. A chicken tracheal swab sample without AIV was used as a negative control. The procedure for the detection of chicken swab samples was the same as that for the inactivated AIV H5N1. Triplicate tests were conducted for each measured titer in the range from $2^{-4}$ to $2^{0} \mathrm{HAU} / 50 \mu \mathrm{l}$. As shown in Fig. $4 \mathrm{~b}$, the frequency change of the chicken tracheal swab that was experimentally spiked with the AIV H5N1 was comparable to the stock AIV H5N1 in the corresponding HAU titers. A linear relationship between the frequency shift and the $\log _{2}$ value of the AIV H5N1 titer was obtained, and the correlation coefficient was 0.95 . These test results demonstrated that the nanowell-based aptasensor was able to detect AIV H5N1 in chicken tracheal swab samples. However, larger error bars were observed in the detection of swab samples as opposed to the stock virus sample. This is possibly due to the interference/noise of impurities presented in the tracheal swab samples on the detection signal.

A comparison study between the nanowell-based QCM aptasensor and other reported QCM immunosensors/aptasensors for detection of AIVs is summarized in Table S1 (Supplementary Materials). As can be seen, the developed nanowell-based QCM aptasensor has 
acceptable sensitivity and is advantageous in label-free and rapid detection with a detection time of only $10 \mathrm{~min}$. It appears to be an efficient technique for AIV detection.

\section{Conclusions}

In this study, a nanowell-based electrode was prepared using a cost-effective method and then was employed for the development of a nanowell-based QCM aptasensor for rapid, sensitive, and label-free detection of AIV H5N1. The use of a nanowell-based electrode effectively increased the immobilization capacity of aptamers, which resulted in a fivefold enhancement in amount of aptamer immobilization compared to the one without the nanowells. The performance of the nanowell-based QCM aptasensor was evaluated and studied by detection of both stock AIV H5N1 and chicken tracheal swab samples experimentally spiked with AIV H5N1. Results showed that the developed nanowell-based QCM aptasensor dramatically reduced detection time down to $10 \mathrm{~min}$. A linear quantitative relationship was obtained between the measured frequency change and the titers of AIV H5N1 in both of the ranges of $2^{-4}-2^{0}$ HAU/50 $\mu \mathrm{l}$, and $2^{0}-2^{4} \mathrm{HAU} / 50 \mu \mathrm{l}$, which enabled the aptasensor to enumerate the target virus. The detection limit was found to be $2^{-4} \mathrm{HAU} / 50 \mu \mathrm{l}$ for the stock AIV H5N1 and $2^{-3} \mathrm{HAU} / 50 \mu \mathrm{l}$ for the experimental positive chicken tracheal swab sample. No interference was observed from nontarget AIV subtypes of H1N1, H2N2, H7N2, and H5N3. In comparison with other reported QCM immunosensors/aptasensors for detection of AIVs, the developed nanowell-based aptasensor reduced the detection time with a label-free assay. It is potentially a simple, rapid, sensitive, and label-free technique for virus detection.

\section{Acknowledgments}


This research was supported in part by U.S Department of Agriculture/National Institute

of Food and Agriculture (project \# 20093560305063) and Arkansas Biosciences Institute (project \# 0383-43076-24-2333).

\section{References}

[1] B. Zhang, D. Tang, B. Liu, H. Chen, Y. Cui and G. Chen, GoldMag nanocompositefunctionalized graphene sensing platform for one-step electrochemical immunoassay of alpha-fetoprotein, Biosens. Bioelectron. 28 (2011) 174-180.

[2] I. Ojeda, B. Garcinuño, M. Moreno-Guzmán, A. González-Cortés, M. Yudasaka, S. Iijima, F. Langa, P. Yáñez-Sedeño and J. M. Pingarrón, Carbon nanohorns as a scaffold for the construction of disposable electrochemical immunosensing platforms. Application to the determination of fibrinogen in human plasma and urine, Anal. Chem. 86 (2014) 7749-7756.

[3] J. Liu, J. Wang, T. Wang, D. Li, F. Xi, J. Wang and E. Wang, Three-dimensional electrochemical immunosensor for sensitive detection of carcinoembryonic antigen based on monolithic and macroporous graphene foam, Biosens. Bioelectron. 65 (2014) 281-286.

[4] C. Li, Y. Su, S. Zhang, X. Lv, H. Xia and Y. Wang, An improved sensitivity nonenzymatic glucose biosensor based on a $\mathrm{Cu}_{x} \mathrm{O}$ modified electrode, Biosens. Bioelectron. 26 (2010) 903-907.

[5] K. K. Lee, P. Y. Loh, C. H. Sow and W. S. Chin, Nanocomposite of $\mathrm{MoS}_{2}$ on ordered mesoporous carbon nanospheres: A highly active catalyst for electrochemical hydrogen evolution, Electrochem. Commun. 20 (2012) 128-132.

[6] Y. Song, X. Lu, Y. Li, Q. Guo, S. Chen, L. Mao, H. Hou and L. Wang, Nitrogen-doped carbon nanotubes supported by macroporous carbon as an efficient enzymatic biosensing platform for glucose, Anal. Chem. 88(2) (2016) 1371-1377.

[7] A. Dahlin, M. Zach, T. Rindzevicius, M. Kall, D. S. Sutherland and F. Hook, Localized surface plasmon resonance sensing of lipid-membrane-mediated biorecognition events, $J$. Am. Chem. Soc. 127 (2005) 5043-5048.

[8] H. Y. Lee, J. W. Park, J. M. Kim, H. S. Jung and T. Kawai, Well-oriented nanowell array metrics for integrated digital nanobiosensors, Appl. Phys. Lett. 89 (2006) 113901.

[9] B. Jung and W. Frey, Fabrication and localized surface plasmon properties of triangular gold nanowell arrays in a glass substrate, J Nanosci Nanotechnol. 15(1) (2015) 688-692. 
[10] L. A. Wollenberg, J. E. Jett, Y. Wu, D. R. Flora, N. Wu, T. S. Tracy and P. M. Gannett, Selective filling of nanowells in nanowell arrays fabricated using polystyrene nanosphere lithography with cytochrome P450 enzymes, Nanotechnology. 23(38) (2012) 385101.

[11] A. J. Torres, A. S. Hill and J. C. Love, Nanowell-based immunoassays for measuring singlecell secretion: characterization of transport and surface binding, Anal. Chem. 86(23) (2014) 11562-11569.

[12] M. S. Cha, J. K. Lee, S. H. Cho, J. G. Park, H. Y. Lee, S. H. Lim and Y. R. Yoon, Quantitative analysis of H5N1 DNA hybridization on nanowell array electrode, J Nanosci Nanotechnol. 13(8) (2013) 5245-5249.

[13] A. Burns, D. van der Mensbrugghe and H. Timmer. World Bank report [Internet]. 2008. Available from:

http://siteresources.worldbank.org/EXTAVIANFLU/Resources/EvaluatingAHIeconomics_2 008.pdf (Accessed: 2016-04-26)

[14] WHO. Cumulative number of confirmed human cases of avian influenza A (H5N1) reported to WHO, 2003-2016 [Internet]. 2016. Available from:

http://www.who.int/influenza/human_animal_interface/EN_GIP_20160404cumulativenumbe rH5N1cases.pdf (Accessed: 2016-04-26)

[15] D. S. Leland and C. C. Ginocchio, Role of cell culture for virus detection in the age of technology, Clin. Microbiol. Rev. 2007, 20, 49-78.

[16] H. T. Ho, H. L. Qian, F. He, T. Meng, M. Szyporta, N. Prabhu, M. Prabakaran, K. P. Chan, and J. Kwang, Rapid detection of H5N1 subtype influenza viruses by antigen capture enzyme-linked immunosorbent assay using H5- and N1-specific monoclonal antibodies, Clin. Vaccine. Immunol. 16 (2009) 726-732.

[17] E. K. Ng, P. K. Cheng, A. Y. Ng, T. L. Hoang and W. W. Lim, Influenza A H5N1 detection, Emerg. Infect. Dis. 11 (2005) 1303-1305.

[18] H. Bai, R. Wang, B. Hargis, H. Lu and Y. Li, A SPR aptasensor for detection of avian influenza virus H5N1, Sensors 12(9) (2012) 12506-12518.

[19] K. Nomura, S. C. Gopinath, T. Lakshmipriya, N. Fukuda, X. Wang and M. Fujimaki, An angular fluidic channel for prism-free surface-plasmon-assisted fluorescence capturing, Nat Commun 4 (2013) 2855.

[20] S. C. Gopinath, K. Awazu and M. Fujimaki, Detection of influenza viruses by a waveguidemode sensor, Anal. Methods 2 (2010) 1880-1884. 
[21] Y. Pang, Z. Rong, J. Wang, R. Xiao and S. Wang, A fluorescent aptasensor for H5N1 influenza virus detection based-on the core-shell nanoparticles metal-enhanced fluorescence (MEF), Biosens Bioelectron. 66 (2015) 527-532.

[22] Y. Li, M. Hong, B. Qiu, Z. Lin, Y. Chen, Z. Cai and G. Chen, Highly sensitive fluorescent immunosensor for detection of influenza virus based on $\mathrm{Ag}$ autocatalysis, Biosens Bioelectron 54 (2014) 358-364.

[23] R. Singh, A. Sharma, S. Hong and J. Jang, Electrical immunosensor based on dielectrophoretically-deposited carbon nanotubes for detection of influenza virus H1N1, Analyst 139(21) (2014) 5415-5421.

[24] Z. Xie, J. Huang, S. Luo, Z. Xie, L. Xie, J. Liu, Y. Pang, X. Deng and Q. Fan, Ultrasensitive electrochemical immunoassay for avian influenza subtype $\mathrm{H} 5$ using nanocomposite, PLoS One 9(4) (2014) e94685.

[25] J. Lin, R. Wang, P. Jiao, Y. Li, Y. Li, M. Liao, Y. Yu and M. Wang, An impedance immunosensor based on low-cost microelectrodes and specific monoclonal antibodies for rapid detection of avian influenza virus H5N1 in chicken swabs, Biosens Bioelectron 67 (2015) 546-552.

[26] R. Wang, Y. Wang, K. Lassiter, Y. Li, B. Hargis, S. Tung, L. Berghman and W. Bottje, Interdigitated array microelectrode based impedance immunosensor for detection of avian influenza virus H5N1, Talanta 79(2) (2009) 159-164.

[27] R. Wang, L. Xu and Y. Li, Bio-nanogate controlled enzymatic reaction for virus sensing, Biosens Bioelectron 67 (2015) 400-407.

[28] T. W. Owen, R. O. Al-Kaysi, C. J. Bardeen and Q. Cheng, Microgravimetric immunosensor for direct detection of aerosolized influenza A virus particles, Sensors and Actuators B: Chemical 126 (2007) 691-699.

[29] T. M. Peduru-Hewa, G. A. Tannock, D. E. Mainwaring, S. Harrison and J. V. Fecondo, The detection of influenza A and B viruses in clinical specimens using a quartz crystal microbalance, J Virol Methods. 162(1-2) (2009) 14-21.

[30] D. Li, J. Wang, R. Wang, Y. Li, D. Abi-Ghanem, L. Berghman, B. Hargis and H. Lu, A nanobeads amplified QCM immunosensor for the detection of avian influenza virus H5N1, Biosens Bioelectron. 26(10) (2011) 4146-4154.

[31] L. Brockman, R. Wang, J. Lum and Y. Li, QCM aptasensor for rapid and specific detection of avian influenza virus, O. J. Appl. Biosens. 2 (2013) 97-103.

[32] R. Wang and Y. Li, Hydrogel based QCM aptasensor for detection of avian influenza virus, Biosens Bioelectron. 42 (2013) 148-155. 
[33] Y. Ding and J. Erlebacher, Nanoporous metals with controlled multimodal pore size distribution, J Am Chem Soc. 125(26) (2003) 7772-7773.

[34] X. Hu, R. Wang, Y. Ding, X. Zhang and W. Jin, Electrochemiluminescence of CdTe quantum dots as labels at nanoporous gold leaf electrodes for ultrasensitive DNA analysis, Talanta 80 (2010) 1737-1743.

[35] D. Qu, B-C. Kim, C-WJ. Lee, M. Ito, H. Noguchi and K. Uosaki, 1,6-Hexanedithiol selfassembled monolayers on $\mathrm{Au}(111)$ investigated by electrochemical, spectroscopic, and molecular mechanics methods, J. Phys. Chem. C 114 (2010) 497-505.

[36] S. Chah, J. H. Fendler and J. Yi, In-situ analysis of step-wise self-assembled 1,6 Hexanedithiol multilayers by surface plasmon resonance measurements, Chem. Commun. 18 (2002) 2094-2095.

[37] M. Chirea, Electron transfer at gold nanostar assemblies: A study of shape stability and surface density influence, Catalysts 3 (2013) 288-309.

[38] H. Zhang, M. Liu, T. Zhou, B. Dong and C. Y. Li, Stepwise assembly of a cross-linked freestanding nanoparticle sheet with controllable shape, Nanoscale. 7(25) (2015) 11033-11039.

[39] S. Tombelli, M. Mascini and A. F. P. Turner, Improved procedures for immobilisation of oligonucleotides on gold-coated piezoelectric quartz crystals, Biosens. Bioelectron. 17 (2002) 929-936.

[40] R. A. Lamb and R. M. Krug. Orthomyxoviridae. In: D. M. Knipe and P. M. Howley, editor. In Field Virology. Philadelphia, PA: Lippincott William \& Wilkins Publisher; 2001. p. 1487-1531.

[41] R. Wang, J. Zhao, T. Jiang, Y. M. Kwon, H. Lu, P. Jiao, M. Liao and Y. Li, Selection and characterization of DNA aptamers for use in detection of avian influenza virus H5N1, J Virol Methods 189(2) (2013) 362-369.

[42] Y. R. Jian, S. Y. Chang, P. Y. Lin, Y. H. Yang and Y. H. Chuang, Inactivated influenza virus vaccine is efficient and reduces IL-4 and IL-6 in allergic asthma mice, Influenza Other Respir Viruses 7(6) (2013) 1210-1217. 


\section{Table 1}

Comparison between QCM aptasensors with and without nanowells for detection of H5N1 AIV

\begin{tabular}{llllll}
\hline QCM aptasensor & \multicolumn{5}{l}{ Frequency Change (Hz), Mean \pm S.D. } \\
\cline { 2 - 6 } & \multicolumn{4}{l}{ H5N1 titer in HAU } & \\
\cline { 2 - 6 } & $2^{-4}$ & $2^{-2}$ & $2^{0}$ & $2^{2}$ & $2^{4}$ \\
\hline With nanowells & $-10 \pm 2$ & $-18 \pm 3$ & $-38 \pm 4$ & $-126 \pm 10$ & $-259 \pm 22$ \\
Without nanowells & $-7 \pm 3$ & $-13 \pm 1$ & $-30 \pm 6$ & $-72 \pm 11$ & $-163 \pm 24$ \\
$\begin{array}{l}P \text {-value between the QCM aptasensor } \\
\text { with and without nanowells }\end{array}$ & 0.11 & 0.03 & 0.05 & $<0.01$ & $<0.01$ \\
\hline
\end{tabular}

*Three replicates 


\section{Figure Legends}

Figure 1. (a) Chemical modification of the nanoporous gold film onto the gold electrode surface to form a nanowell-based electrode. (b) The SEM image of a nanowell-based electrode surface. (c) Pictures of the QCM electrode before (I) and after (II) the nanoporous gold film modification.

Figure 2. (a) Schematic diagram for the fabrication of the nanowell-based QCM aptasensor using the $\mathrm{NH}_{2}$-aptamer. (b) The typical temporal responses of frequency shifts for the stepwise assembly of the fabrication of the nanowell-based QCM aptasensor: (1) SAM formed on the sensor in equilibrium with deionized water; (2) NHS/EDC activation; (3) $\mathrm{NH}_{2}$-aptamer immobilization; and (4) wash to get the baseline.

Figure 3. (a) The typical QCM detection curve of AIV H5N1 at the titers of 2 and 8 HAU. (b) Results of AIV H5N1 detection in the range from $2^{-4}$ to $2^{4}$ HAU using the nanowell-based QCM aptasensor. The means and error bars (standard deviations) were calculated based on 3 replicates. (c) SEM images of the top view of the binding of a H5N1 virus on the surface of a nanowell-based QCM electrode.

Figure 4. (a) Frequency shifts of the nanowell-based QCM aptasensor for the target H5N1 AIV along with the four non-target AIV subtypes, each at 2 HAU. Error bars indicate standard deviation $(n=3)$. (b) Frequency shifts caused by negative controls and tracheal swab samples spiked with H5N1 AIV ranged from $2^{-4}$ to $2^{0}$ HAU. The means and error bars (standard deviation) were calculated based on 3 replicates. 



\section{Figure 1}
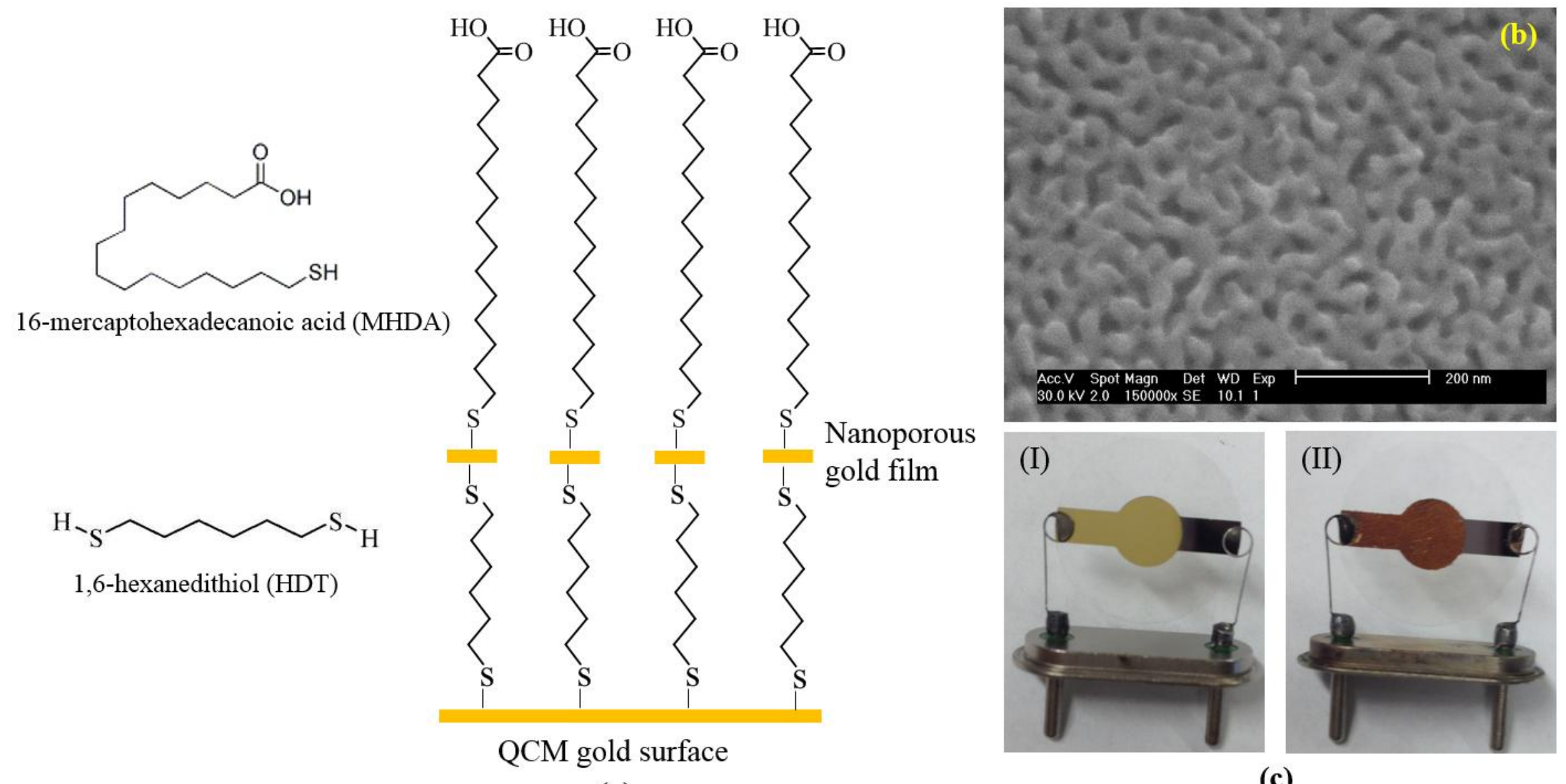

(a)

(c) 


\section{Figure 2}

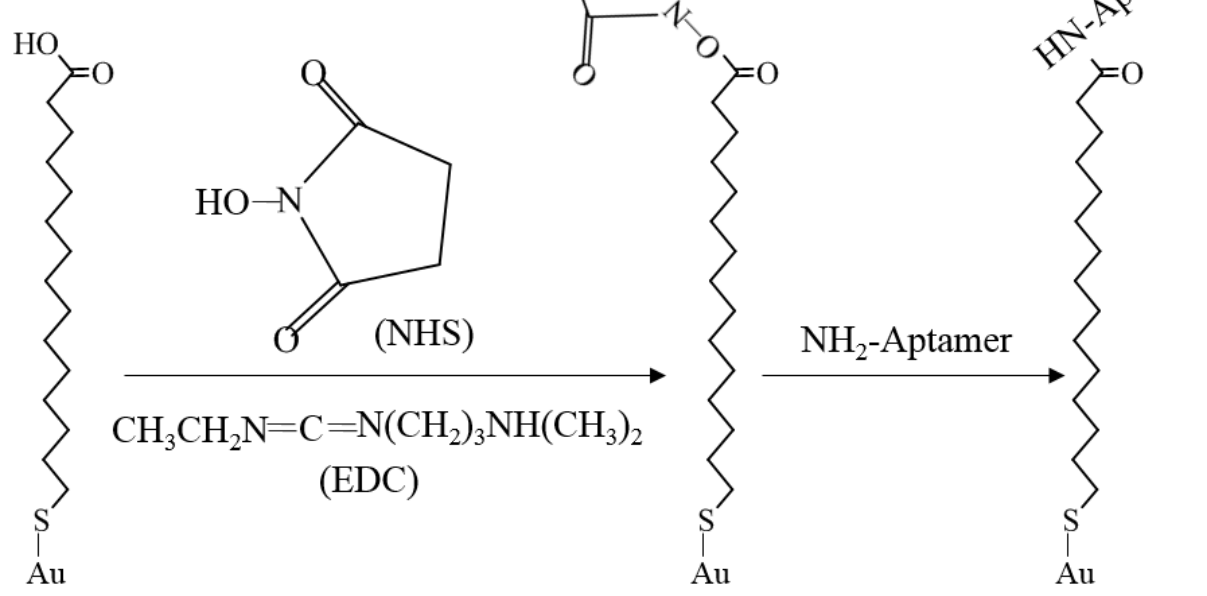

(a)

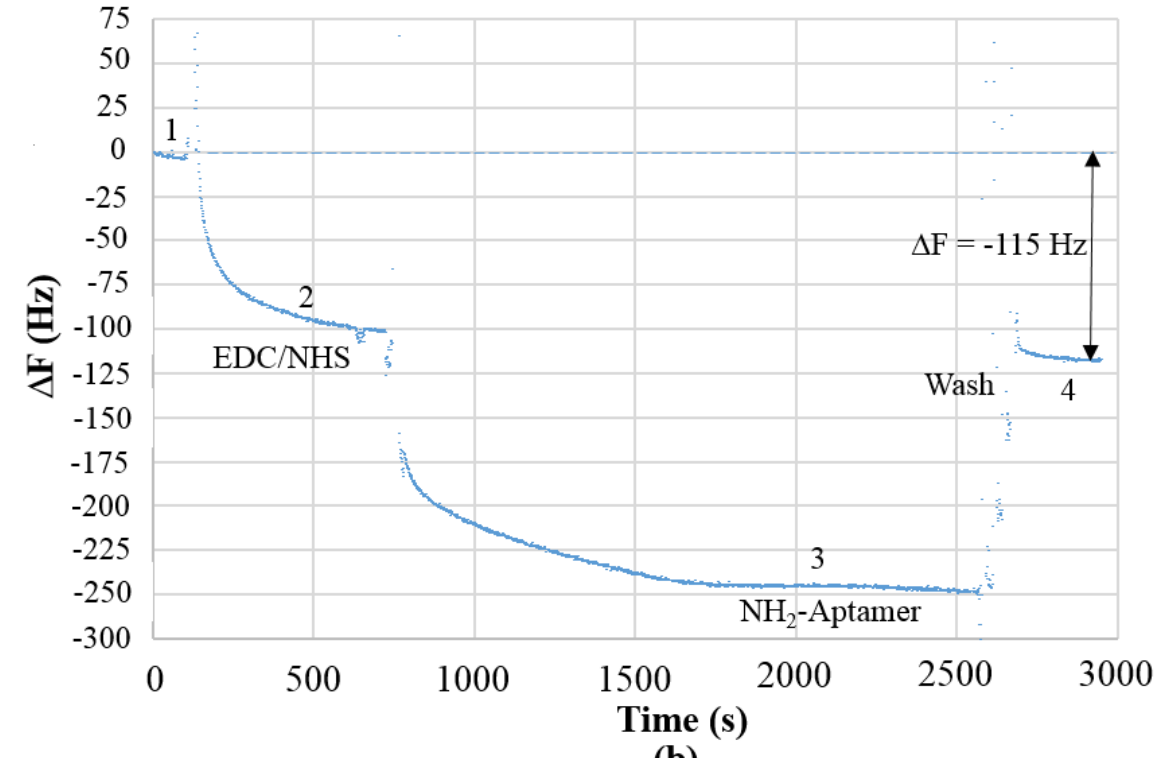

(b) 


\section{Figure 3}

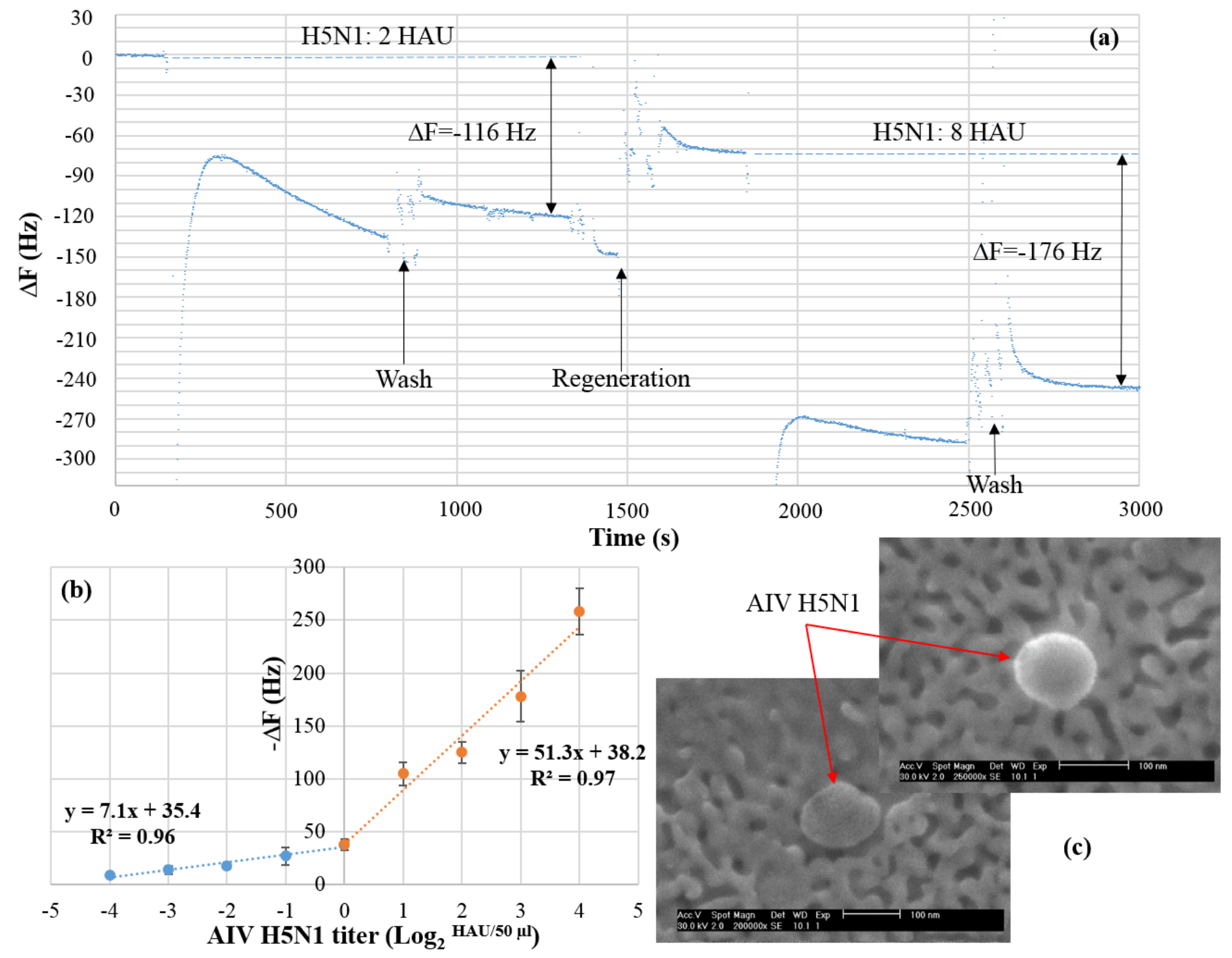




\section{Figure 4}

140

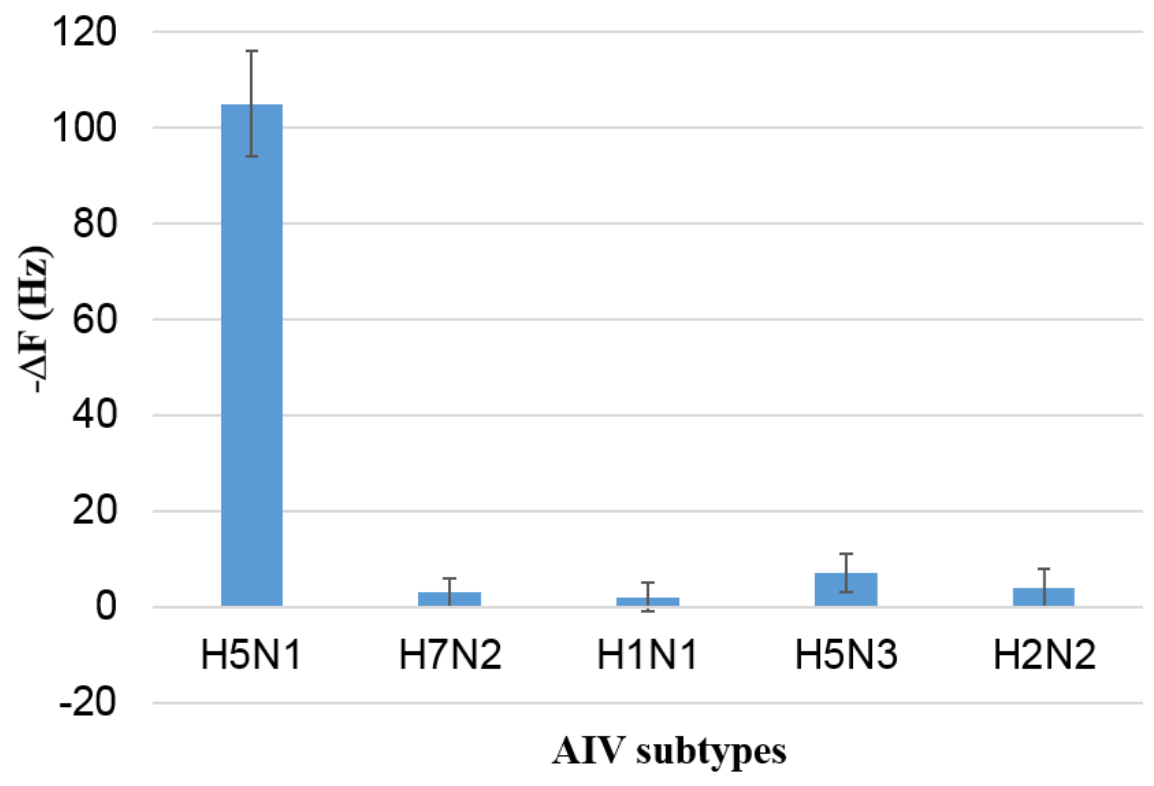

60

(b)

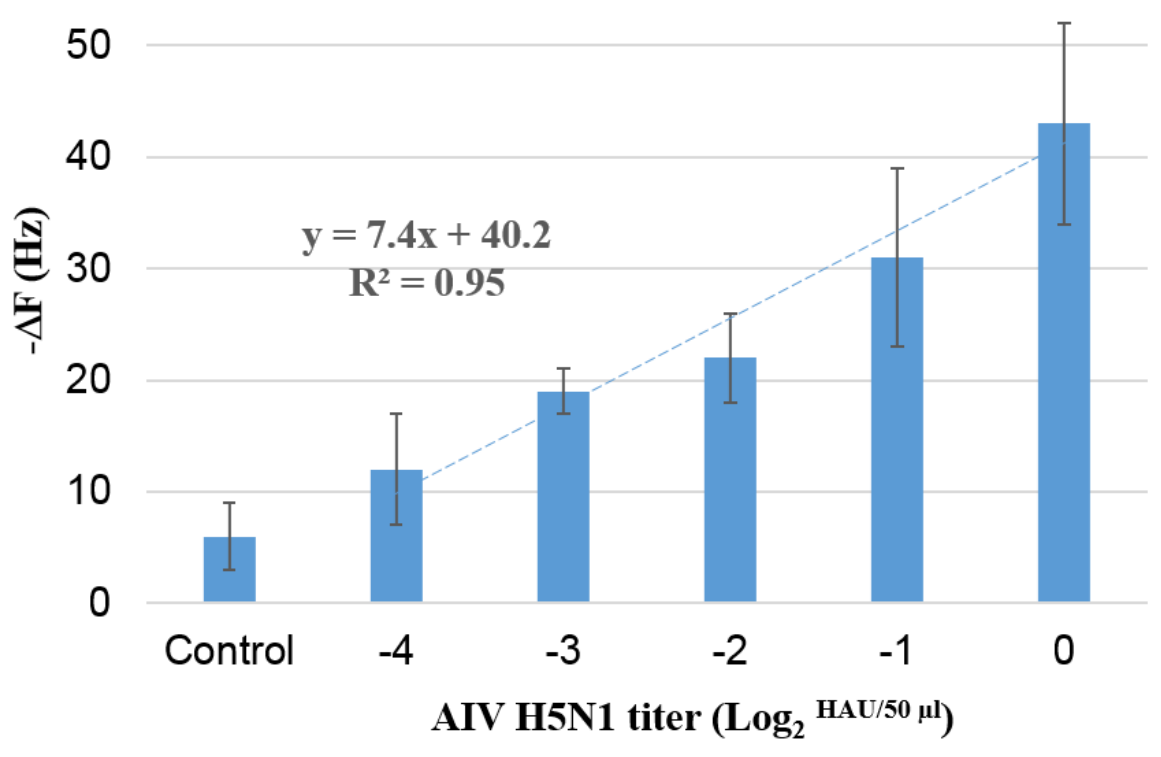

